\title{
Microvasculature Features of Vogt-Koyanagi-Harada Disease Revealed by Widefield Swept-Source Optical Coherence Tomography Angiography
}

OPEN ACCESS

Edited by:

Hetian Lei,

Shenzhen Eye Hospital, China

Reviewed by:

Han Zhang,

The First Affiliated Hospital of China

Medical University, China

Qianli Meng,

Guangdong Provincial People's

Hospital, China

Xiaorong $\mathrm{Li}$

Tianjin Medical University Eye

Hospital, China

${ }^{*}$ Correspondence:

Jin Yuan

yuanjincornea@126.com

Feng Wen

wenfeng208@foxmail.com

Wei Chi

chiwei@mail.sysu.edu.cn

tThese authors have contributed equally to this work

Specialty section:

This article was submitted to

Ophthalmology,

a section of the journal

Frontiers in Medicine

Received: 02 June 2021 Accepted: 16 September 2021

Published: 14 October 2021

Citation:

Ye X, Zhang H, Xiao P, Wang G, Hu X, Yan C, Li F, Hu Y, Su L, Luo J, Yuan J,

Wen F and Chi W (2021)

Microvasculature Features of

Vogt-Koyanagi-Harada Disease

Revealed by Widefield Swept-Source

Optical Coherence Tomography

Angiography. Front. Med. 8:719593.

doi: 10.3389/fmed.2021.719593
Xiaoyuan Ye ${ }^{1 \dagger}$, Haiping Zhang ${ }^{2,3+}$, Peng Xiao ${ }^{1}$, Gengyuan Wang ${ }^{1}$, Xiaoqing $\mathrm{Hu}^{1}$, Chun Yan ${ }^{1}$, Fan $\mathrm{Li}^{1}$, Yixin $\mathrm{Hu}^{1}$, Lishi Su ${ }^{1}$, Jiawen Luo ${ }^{1}$, Jin Yuan ${ }^{1 *}$, Feng Wen ${ }^{1 *}$ and Wei Chi ${ }^{1 *}$

${ }^{1}$ State Key Laboratory of Ophthalmology, Zhongshan Ophthalmic Center, Sun Yat-sen University, Guangzhou, China, ${ }^{2}$ Tianjin Aier Eye Hospital, Tianjin, China, ${ }^{3}$ Aier Eye Institute, Changsha, China

Background: Vogt-Koyanagi-Harada (VKH) disease is a multisystem autoimmune disorder which could induce bilateral panuveitis involving the posterior pole and peripheral fundus. Optical coherence tomography angiography (OCTA) provides several advantages over traditional fluorescence angiography for revealing pathological abnormalities of the retinal vasculature. Until recently, however, the OCTA field of view (FOV) was limited to $6 \times 6 \mathrm{~mm}^{2}$ scans.

Purpose: This study examined retinal vasculature and choriocapillaris abnormalities across multiple regions of the retina $\left(15 \times 9 \mathrm{~mm}^{2}\right.$ wide field, macular, peripapillary regions) among acute and convalescent VKH patients using a novel widefield swept-source OCTA (WSS-OCTA) device and assessed correlations between imaging features and best-corrected visual acuity (BCVA).

Methods: Twenty eyes of 13 VHK disease patients in the acute phase, 30 eyes of 17 patients in the convalescent phase, and 30 eyes of 15 healthy controls (HCs) were included in this study. Vascular length density (VLD) in superficial and deep vascular plexuses (SVP, DVP), vascular perfusion density (VPD) in SVP, DVP, and choriocapillaris (CC), and flow voids (FV) in CC were measured across multiple retinal regions via WSS-OCTA (PLEX Elite 9000, Carl Zeiss Meditec Inc., USA) using the $15 \times 9 \mathrm{~mm}^{2}$ scan pattern centered on the fovea and quantified by ImageJ.

Results: Compared to HCs, acute phase VKH patients exhibited significantly reduced SVP-VLD, SVP-VPD, and CC-VPD across multiple retinal regions (all $p<0.01$ ). Notably, the FV area was more extensive in $\mathrm{VKH}$ patients, especially those in the acute phase $(p<0.01)$. These changes were reversed in the convalescent phase. Stepwise multiple linear regression analysis demonstrated that macular DVP-VLD and macular CC-VPD were the best predictive factors for BCVA in the acute and convalescent VKH groups.

Conclusion: The wider field of SS-OCAT provides more comprehensive and detailed images of the microvasculature abnormalities characterizing $\mathrm{VKH}$ disease. 
The quantifiable and layer-specific information from OCTA allows for the identification of sensitive and specific imaging markers for prognosis and treatment guidance, highlighting WSS-OCTA as a promising modality for the clinical management of VKH disease.

Keywords: Vogt-Koyanagi-Harada disease, widefield swept-source optical coherence tomography angiography, vascular length density, vascular perfusion density, flow voids

\section{INTRODUCTION}

Vogt-Koyanagi-Harada (VKH) disease is a multisystemic autoimmune disorder primarily afflicting pigmented tissues. In Asia, VKH disease is a relatively common vision-threatening disorder (1).The classic clinical characteristics are bilateral panuveitis, hypoacusis, meningitis, and cutaneous involvement such as poliosis, vitiligo, and alopecia (2). While the precise mechanisms underlying targeted pigmented tissue attack are still uncertain, it is likely that aberrant $\mathrm{T}$ cell-mediated inflammation contributes to disease initiation and maintenance $(3,4)$. Ophthalmic manifestations could involve the choroidal stroma, retinal pigment epithelium, and outer retina at the posterior pole and peripheral fundus. Inflammation of the choroidal stroma and retinal pigment epithelium layer (RPE) results in a series of changes, including choroidal depigmentation, sunset glow fundus (SGF), subretinal neovascularization, and even exudative retinal detachment (5-7). The clinical course of ophthalmic manifestations can be divided into four stages, prodromal, uveitic, chronic, and chronic recurrent, according to findings from multimodal ocular vascular imaging, traditional indocyanine green angiography (ICGA), fluorescein angiography (FA), and optical coherence tomography (OCT) (5). Injection of fluorescent dye into the circulation reveals characteristic vascular patterns that provide clues to disease progression (8). However, fluorescent imaging modalities (ICGA and FA) do not allow for quantitative analysis of retinal and choroidal blood flow characteristics. Moreover, there are inherent risks from intravenous administration of these dyes.

Optical coherence tomography angiography (OCTA) permits non-invasive, detailed, and depth-resolved imaging of the chorioretinal microvasculature in disease states such as diabetic retinopathy, age-related macular degeneration, retinal vein occlusion, and uveitis (9-15). In addition, several OCTA studies have documented changes in the chorioretinal microvasculature associated with $\mathrm{VKH}$ disease (16-24). However, traditional OCTA images provide a limited field-of-view, necessitating montage imaging. Further, the aforementioned OCTA studies analyzed limited vascular features.

The introduction of swept-source technology to OCTA has substantially expanded the potential field of view (FOV), which is particularly advantageous for disorders such as $\mathrm{VKH}$ disease afflicting broad regions of the retina. In this study, we enrolled $\mathrm{VKH}$ patients in acute and convalescent phases and used the newest PLEX Elite 9000 swept-source OCTA (SS-OCTA) system to capture widefield $\left(15 \times 9 \mathrm{~mm}^{2}\right)$ OCTA images of the chorioretinal microvasculature in macular and peripapillary regions to provide a more detailed description of disease progression and identify signs indicative of visual dysfunction and recovery.

\section{MATERIALS AND METHODS}

\section{Study Population}

This cross-sectional, observational study, included 20 eyes of 13 patients with acute $\mathrm{VKH}$ disease, 30 eyes of $17 \mathrm{VKH}$ disease patients in the convalescent stage, and 30 eyes of 15 age-matched healthy volunteers without any ophthalmological and/or systemic disorders examined at the Zhongshan Ophthalmic Center from November 2019 to December 2020. Patients were diagnosed based on revised diagnostic criteria established by the First International Workshop on Vogt-Koyanagi-Harada (VKH) disease (25), and grouped according to disease stage. Patients initially diagnosed or receiving systemic corticosteroids for $<2$ weeks were considered in the acute stage. Among these patients, those with severe exudative retinal detachment, severe anterior chamber inflammation, or vitreous opacity were excluded, and the rest were allocated to the acute stage group. Alternatively, patients receiving systemic corticosteroids for over 3 months and showing no signs of acute ocular inflammation such as choroiditis, serous retinal detachment, disc edema, or exudative retinal detachment were included in the convalescent stage group.

All procedures were performed in compliance with the tenets of the Declaration of Helsinki, and the study was approved by the Ethics Committee of Zhongshan Ophthalmic Center (Guangzhou, China 2019KYPJ127). Written informed consent was obtained from each participant. The following data were collected for all participants: age, sex, best-corrected visual acuity (BCVA) as measured using a Snellen chart, and bilateral intraocular pressure. Patients also received slit-lamp microscopy, indirect fundus ophthalmoscopy, and FA examinations.

\section{Optical Coherence Tomography Angiography Acquisition}

All widefield swept-source OCTA (WSS-OCTA) images were acquired using a PLEX Elite 9000 SS-OCTA device (Carl Zeiss Meditec Inc., USA) that simultaneously assesses the fundus with a central wavelength of $1,060 \mathrm{~nm}(1,000-1100 \mathrm{~nm}$ full bandwidth) and operates at 100,000 A-scans per second. The system also includes an active eye-tracking system. For each eye, OCTA scans of $15 \times 9 \mathrm{~mm}^{2}$ centered on the fovea were performed after pupil dilation. All images were collected by the same experienced technician $(\mathrm{Hu})$. Images with either substantial motion artifact or incorrect segmentation were excluded. Representative pictures are presented in Figure 1. To 

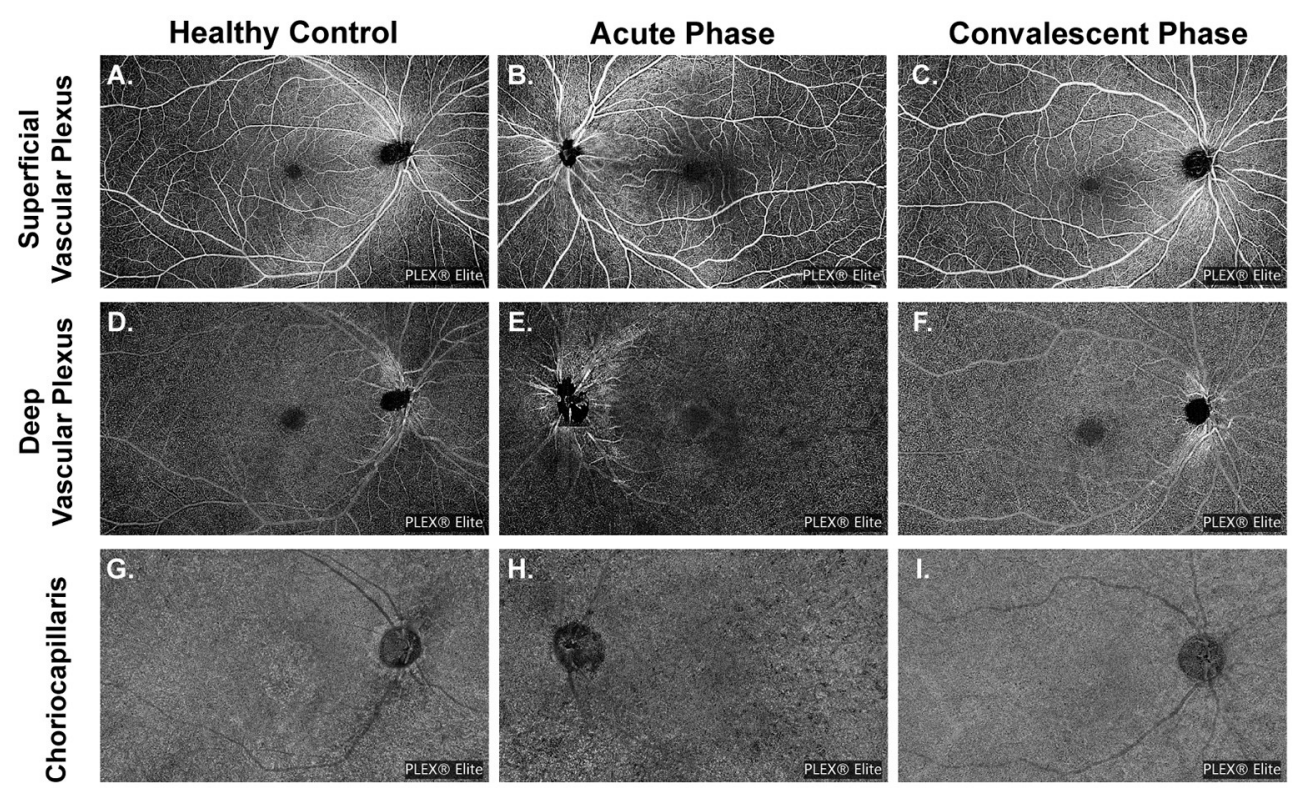

FIGURE 1 | Demonstration of representative images of different slabs in three groups. (A,D,G) Slabs are from a healthy volunteer (No. 14, the right eye). (B,E,H) Slabs are from a patient with acute VKH disease (No. 12, the left eye). (C,F,I) Slabs are from a patient with quiescent VKH disease (No. 17, the right eye).
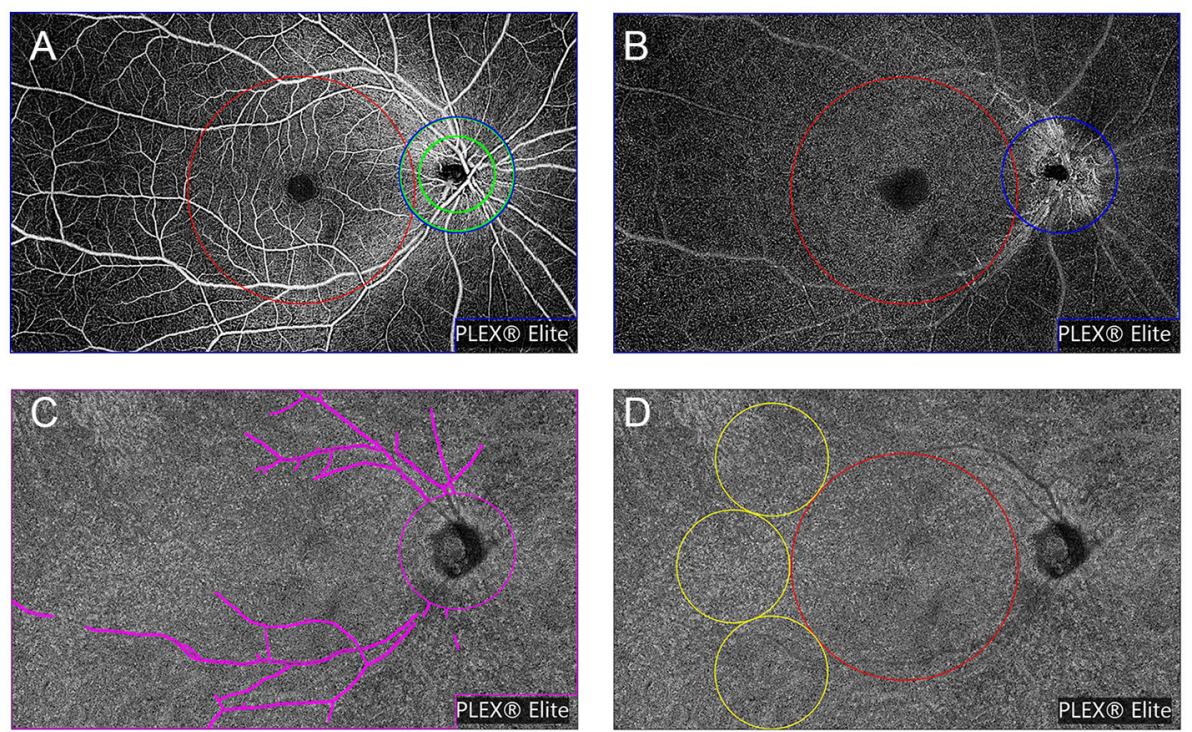

FIGURE 2 | Representation of the regions used to explore OCTA parameters, slabs are from a healthy volunteer (No. 13, the right eye). OCTA parameters were investigated in different regions of the superficial vascular plexus (SVP) slab (A) and deep vascular plexus (DVP) slab (B): (1) the macular region (red circle with a diameter of $6 \mathrm{~mm}$ centered at the fovea); (2) the peripapillary region (a $500 \mu \mathrm{m}$-wide ring of $2 \mathrm{~mm}$ inner diameter and $3 \mathrm{~mm}$ outer diameter centered at the optic nerve head) (green annulus); and (3) the whole field of view (FOV) with optic papilla area (a $3 \mathrm{~mm}$ diameter circle) and the image label "PLEX® Elite" excluded (cropped) (blue polygon shape). (C) Representation of whole FOV removing the large retinal vessel mask used to explore OCTA parameters in choriocapillaris slab (shown in magenta). (D) Representation of regions to explore flow voids metrics: (1) the macular region (shown in red) and (2) the peripheral region (three $3 \mathrm{~mm}-$ diameter circles next to the macular region) (shown in yellow).

quantify the foveal avascular zone (FAZ), vascular length density (VLD), vascular perfusion density (VPD), and flow voids (FV) across regions and layers, all images were first segmented using built-in software. Segmentation lines defining the inner limiting membrane (ILM), inner plexiform layer (IPL), outer plexiform layer (OPL), and RPE were automatically delineated in each Bscan. Any notable segmentation error was manually corrected. Image slabs were generated to reveal the superficial retinal 


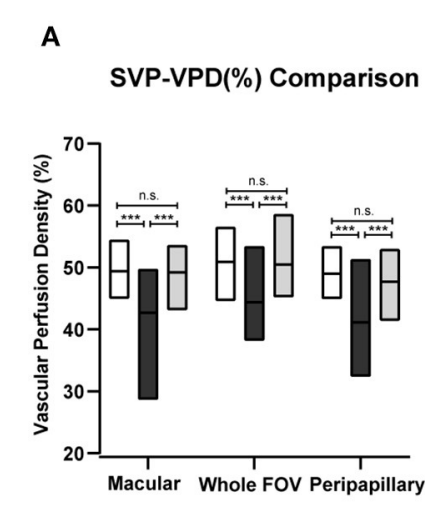

E

CC-VPD(\%) Comparison

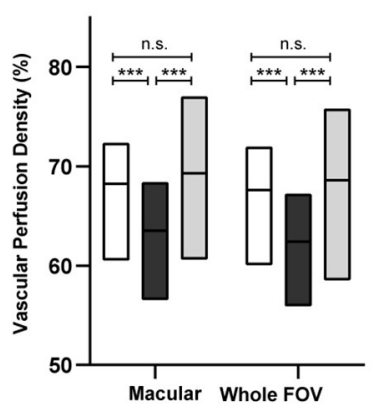

B

DVP-VPD(\%) Comparison

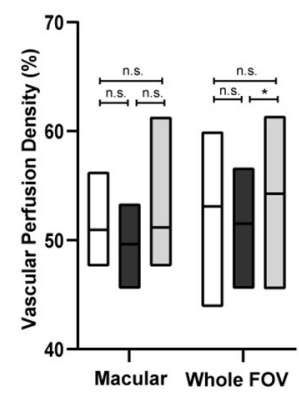

$\mathbf{F}$

CC-FV Comparison

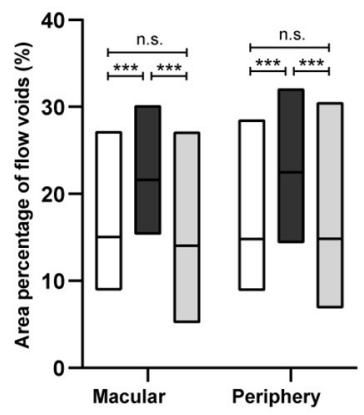

G
C

SVP-VLD $\left(\mathrm{mm}^{-1}\right)$ Comparison DVP-VLD $\left(\mathrm{mm}^{-1}\right)$ Comparison

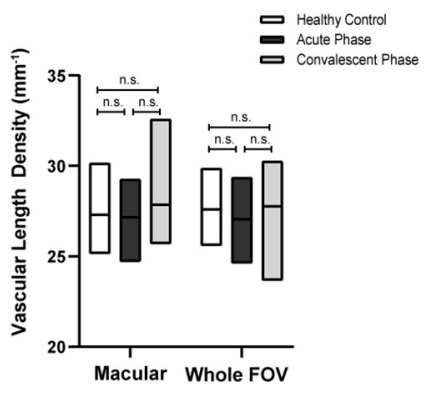

Num. of FV1000 Comparison

H

Average Size of CC-FV Comparison

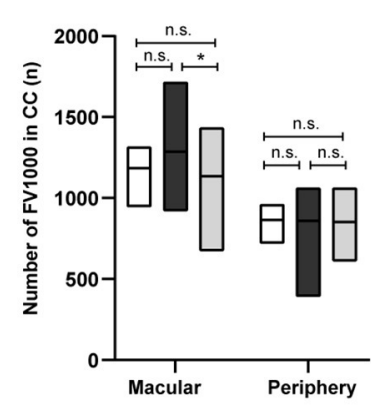

FIGURE 3 | Comparisons of different parameters among three groups in accordance to Tables 2-4. Bar-graphs represent mean (range) (A-F) or median (range) (G,H). $\left({ }^{\star} p<0.05 ;{ }^{* * *} p<0.001\right.$; n.s., non-significant).

vascular plexus (SVP) (from the ILM to the outer boundary of IPL), the deep retinal vascular plexus (DVP) (from the outer boundary of IPL to the outer boundary of OPL), and the choriocapillaris (CC) (derived from a $10 \mu \mathrm{m}$ slab $31-40 \mu \mathrm{m}$ below the RPE) as previously described (26). Projection artifacts caused by the overlying retinal circulation were removed using built-in software.

\section{Image Processing and Measurements of the Parameters}

All images were checked independently by two trained graders (Ye and Zhang). The OCTA slabs were processed and analyzed according to the procedure used in previous works (16, 27-30). Each OCTA slab was loaded into ImageJ (National Institutes of Health, Bethesda, Maryland, USA; https://imagej.net/Welcome) to measure FAZ, VLD or VPD, and FV parameters as follows. The area and perimeter of the FAZ from the SVP slab were measured by two trained graders by manually outlining the FAZ border in ImageJ and measuring the area and length. The Acircularity Index (AI), defined as the ratio of the FAZ perimeter to the perimeter of a circle with equal area (16), was introduced to describe the acircularity of FAZ shape. "Vascular Perfusion Density" is defined as the fraction of area covered by vessels in an enface slab view (more specifically, the ratio of the vessel area to the total region of interest or ROI), and ranges from
0 (no perfusion) to 1 (fully perfused). The procedure for VPD quantification was as follows. First, images were converted to 8bit format. Second, the ROI tool in ImageJ was used to select the target regions for analysis, which included the macular region (a $6 \mathrm{~mm}$ diameter circle centered at the fovea), the peripapillary area (a $500 \mu \mathrm{m}$-wide ring of $2 \mathrm{~mm}$ inner diameter and $3 \mathrm{~mm}$ outer diameter centered at the optic nerve head), and the whole field of view (whole FOV) with optic papilla area (a $3 \mathrm{~mm}$ diameter circle) and the image label "PLEX $®$ Elite" excluded (cropped). Next, images were binarized using commands "Huang's fuzzy" for SVP and DVP slabs or "Phansalkar method" with radius $=15$ for CC slabs (27). Finally, the fractional area of vessels within the ROI (or the perfusion density) was measured. To remove large vessel projection artifacts within the $15 \times 9 \mathrm{~mm}^{2} \mathrm{CC}$ slab, we obtained a large retinal vessel mask from the SVP slab by applying "Default" threshold and then used the Image "wand tool" to select the region representing the large vessels. The superficial retinal vessels were successively masked from the CC slab and then the CC slab was binarized using the "Phansalkar method." Detailed procedures are provided in Figure 2.

"Vascular Length Density" is defined as the vessel length per unit area (31). As far as we know the VPD algorithm is greatly influenced by large blood vessels, and has low sensitivity for detecting capillary changes. Alternatively, the VLD algorithm can more sensitively detect changes in small blood 
TABLE 1 | Demographic and clinical characteristics of study groups.

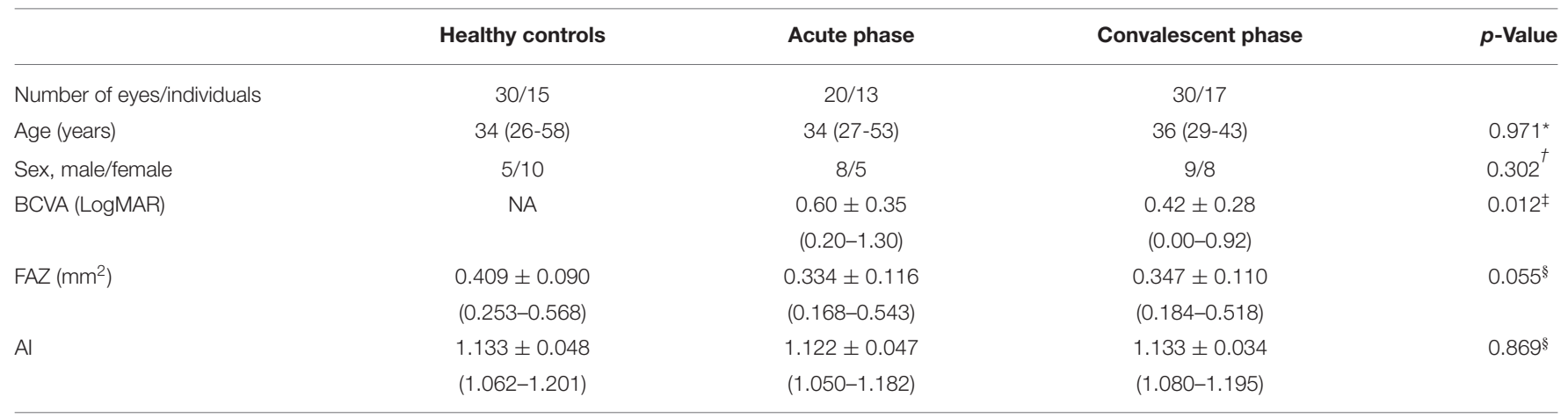

BCVA, best-corrected visual acuity; FAZ, foveal avascular zone; Al, acircularity index. Data are presented as Mean \pm SD (range) or Median (P25-P75), unless otherwise indicated.

*Kuskal-Wallis test.

${ }^{\dagger}$ Chi-square test.

¥Student t-test.

§one-way ANOVA.

TABLE 2 | The vascular perfusion density (VPD) measurements in different slabs and regions.

\begin{tabular}{|c|c|c|c|c|c|c|c|}
\hline VPD (\%) & $\begin{array}{l}\text { Healthy } \\
\text { controls }\end{array}$ & Acute phase & $\begin{array}{c}\text { Convalescent } \\
\text { phase }\end{array}$ & $p$ & $p^{a}$ & $p^{b}$ & $p^{c}$ \\
\hline \multicolumn{8}{|l|}{ SVP } \\
\hline Whole FOV & $\begin{array}{l}50.95 \pm 3.52 \\
(49.64-52.27)\end{array}$ & $\begin{array}{l}44.41 \pm 4.48 \\
(42.31-46.51)\end{array}$ & $\begin{array}{l}50.46 \pm 3.98 \\
(49.35-51.58)\end{array}$ & $<0.001^{*}$ & $<0.001$ & 1.000 & $<0.001$ \\
\hline \multicolumn{8}{|l|}{ DVP } \\
\hline Macular & $\begin{array}{l}50.93 \pm 2.24 \\
(50.10-51.77)\end{array}$ & $\begin{array}{l}49.63 \pm 2.38 \\
(48.52-49.66)\end{array}$ & $\begin{array}{l}51.17 \pm 2.96 \\
(50.10-52.28)\end{array}$ & $0.101^{*}$ & 0.251 & 1.000 & 0.123 \\
\hline Whole FOV & $\begin{array}{l}53.10 \pm 3.59 \\
(51.76-54.44)\end{array}$ & $\begin{array}{l}51.52 \pm 3.38 \\
(49.94-53.10)\end{array}$ & $\begin{array}{l}54.25 \pm 4.32 \\
(52.64-55.86)\end{array}$ & $0.054^{*}$ & 0.474 & 0.746 & 0.048 \\
\hline
\end{tabular}

VPD, vascular perfusion density; SVP, superficial vascular plexus; DVP, deep vascular plexus; CC, choriocapillaris; FOV, field of view. Data are presented as mean \pm SD, (95\%Cl).

"One-Way ANOVA followed by Bonferroni post-hoc test.

${ }^{\dagger}$ Kruskal-Wallis test followed by Bonferroni post-hoc test.

$p^{a}$ : $p$-value between Healthy Controls and Acute VKH patients.

$p^{b}: p$-value between Healthy Controls and VKH patients in convalescent phase.

$p^{c}: p$-value between Acute VKH patients and VKH patients in convalescent phase.

vessels and capillaries. To calculate VLD, we binarized the slab using the "Huang's fuzzy" command and then skeletonized the binary image, followed by measurement of vessel length in the ROI and calculation of VLD as described previously $(31,32)$. Choriocapillaris flow voids (CC-FV), also termed flow deficit in other studies, is defined as the area lacking flow or with flow below the detectable threshold of OCTA $(28,29)$. Flow voids were calculated in the macular region (a $6 \mathrm{~mm}$-diameter circle centered at the fovea) and the peripheral region (three $3 \mathrm{~mm}$ diameter circles next to the macular region). Flow void metrics, including total flow void area fraction (ROI area divided by total flow void area), number of FV, and average size of $\mathrm{FV}$, were calculated for FV area $>1,000 \mu \mathrm{m}^{2}$ (FV1,000) using the "Analyze Particles" function in ImageJ (30).

\section{Statistical Analysis}

All statistical analyses were conducted using SPSS software 22 (SPSS Inc., Chicago, IL). Continuous variables were first tested for normality using the Shapiro-Wilk test and then for homogeneity of variance. Normally distributed variables are presented as mean \pm standard deviation (SD) and variables following a skewed distribution as median and 25 th percentile to 
TABLE 3 | The vascular length density (VLD) measurements in different slabs and regions.

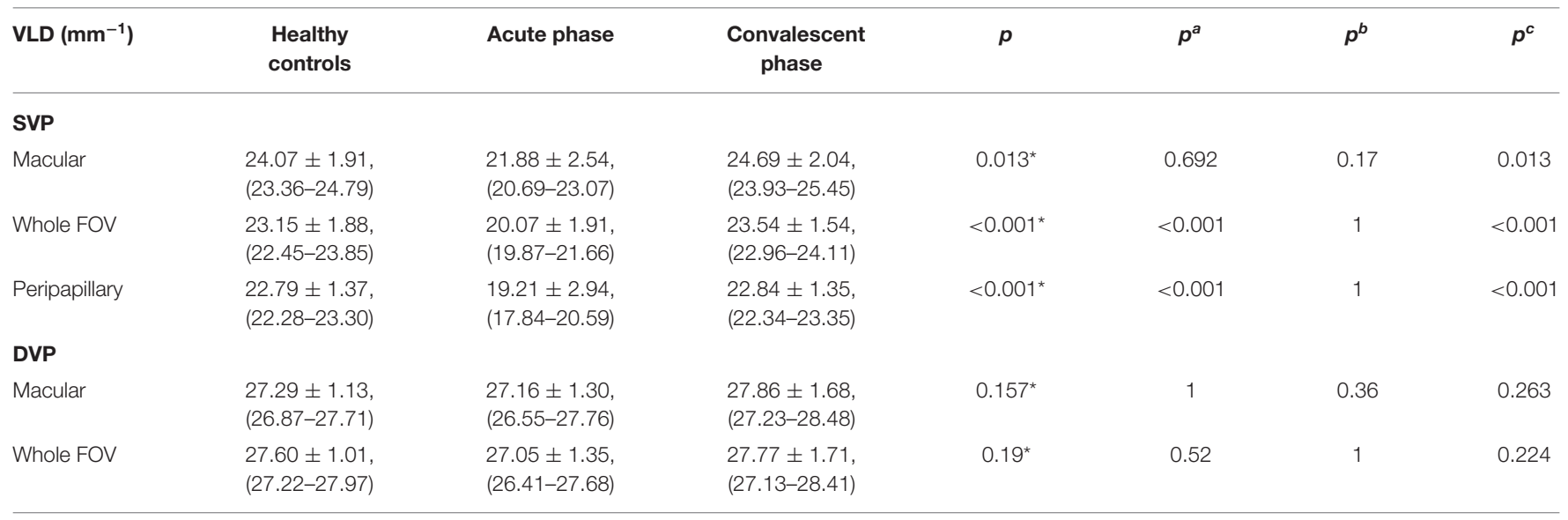

VPD, vascular perfusion density; SVP, superficial vascular plexus; DVP, deep vascular plexus; CC, choriocapillaris; FOV, field of view. Data are presented as mean \pm SD, (95\%Cl).

${ }^{*}$ One-Way ANOVA followed by Bonferroni post-hoc test.

$p^{a}$ : p-value between Healthy Controls and Acute VKH patients.

$p^{b}: p$-value between Healthy Controls and VKH patients in convalescent phase.

$p^{c}: p$-value between Acute VKH patients and VKH patients in convalescent phase.

TABLE 4 | The flow void parameters in CC slab.

\begin{tabular}{|c|c|c|c|c|c|c|c|}
\hline Flow voids & Healthy controls & Acute phase & Convalescent phase & $p$ & $p^{a}$ & $p^{b}$ & $p^{c}$ \\
\hline \multicolumn{8}{|l|}{ Macular $\left(6 \times 6 \mathrm{~mm}^{2}\right)$} \\
\hline Flow void area (\%) & $\begin{array}{l}14.80 \pm 3.84 \\
(13.54-16.54)\end{array}$ & $\begin{array}{l}21.61 \pm 3.80 \\
(19.82-23.39)\end{array}$ & $\begin{array}{l}14.86 \pm 5.87 \\
(11.89-16.19)\end{array}$ & $<0.001^{*}$ & $<0.001$ & 1.000 & $<0.001$ \\
\hline Number of FV1,000 (n) & $\begin{array}{c}1182.00 \\
(1140.25-1246.25)\end{array}$ & $\begin{array}{c}1285.00 \\
(1204.00-1335.00)\end{array}$ & $\begin{array}{c}1211.50 \\
(973.50-1273.25)\end{array}$ & $0.007^{\dagger}$ & 0.092 & 0.635 & 0.004 \\
\hline \multicolumn{8}{|l|}{ Periphery } \\
\hline Flow void area (\%) & $\begin{array}{l}15.04 \pm 4.03 \\
(13.37-16.24)\end{array}$ & $\begin{array}{l}22.49 \pm 4.49 \\
(20.39-24.59)\end{array}$ & $\begin{array}{l}14.04 \pm 5.98 \\
(12.67-18.84)\end{array}$ & $<0.001^{\star}$ & $<0.001$ & 1.000 & $<0.001$ \\
\hline Number of FV1,000 (n) & $\begin{array}{c}868.00 \\
(834.75-921.50)\end{array}$ & $\begin{array}{c}907.50 \\
(835.50-964.25)\end{array}$ & $\begin{array}{c}901.50 \\
(710.50-953.25)\end{array}$ & $0.513^{\dagger}$ & 1.000 & 1.000 & 1.000 \\
\hline
\end{tabular}

FV1,000, flow void area $>1,000 \mu \mathrm{m}^{2}$. Data are presented as mean $\pm S D$, (95\%Cl) or median (P25-P75).

"One-Way ANOVA followed by Bonferroni post-hoc test.

${ }^{\dagger}$ Kruskal-Wallis test followed by Bonferroni post-hoc test.

$p^{a}$ : p-value between healthy controls and acute VKH patients.

$p^{b}: p$-value between healthy controls and VKH patients in convalescent phase.

$p^{c}: p$-value between acute VKH patients and VKH patients in convalescent phase.

75th percentile range (P25-P75). Means of parametric datasets were compared by one-way analysis of variance (ANOVA) and non-parametric datasets by the Kruskal-Wallis test, followed by post-hoc Bonferroni test or Student $t$-test for pair-wise comparisons. Correlations between parametric variables and $\log$ MAR BCVA were analyzed using Pearson Correlation analysis. Stepwise multiple linear regression analysis was run to detect the significant predictors of BCVA. A $p<0.05$ was considered statistically significant for all tests.

\section{RESULTS}

\section{Study Population Characteristics}

Twenty eyes of 13 patients in the acute phase of $\mathrm{VKH}$ disease, 30 eyes of 17 patients in the convalescent phase of $\mathrm{VKH}$ disease, and 30 eyes of 15 age-matched healthy controls (HCs) were included in this study. Images of 10 eyes with $\mathrm{VKH}$ disease were excluded due to significant motion artifacts or incorrect segmentation. Best-corrected visual acuity of the HCs was not 
available. The demographic and clinical characteristics of study groups are summarized in Table $\mathbf{1}$.

\section{Widefield OCTA Parameters of Each Group FAZ and AI}

The FAZ was smaller in eyes of patients with acute or convalescent VKH disease compared to control eyes, while AI was smaller in acute VKH disease eyes than convalescent VKH disease or control eyes. However, neither difference reached statistical significance $(p=0.055$ and 0.0869 , respectively) (Table $\mathbf{1}$ ).

\section{Vascular Perfusion Density and Vascular Length Density}

The VPD of the SVP (SVP-VPD) was significantly lower in the peripapillary region, macular region, and whole FOV of acute VKH disease patients compared to HCs and convalescent VKH disease patients (all $p<0.001$ ). The same tendency was observed in CC and DVP slabs of the macular region and whole FOV scans. The CC-VPD was also significantly lower in acute VKH disease patients compared to convalescent patients and HCs (both $p<0.001$ ). DVP-VPD was also lower in acute phase patients compared to convalescent patients within whole FOV ( $p$ $<0.05$ ). Compared to HCs, convalescent VKH patients exhibited slightly decreased SVP-VPD and modest increased CC-VPD and DVP-VPD in multiple regions but without statistical significance (Table 2) and (Figures 3A,B). Group differences in VLD roughly mirrored those of VPD, albeit with a few notable differences. In contrast to VPD differences between HCs and convalescent VKH patients, the SVP-VLD of convalescent VKH patients was slightly greater in three regions compared to HCs, but again without reaching statistical significance (Table 3) and (Figures 3C,D).

\section{Flow Voids in Choriocapillaris}

Acute-stage VKH patients exhibited significantly larger FV area fractions and average FV sizes in both macular and peripheral retinal regions compared to convalescent-stage patients and HCs (all $p<0.001$ ). Compared to HCs, convalescent-stage patients exhibited a smaller FV area fraction but larger numbers of FV1,000 (single flow void area $>1000 \mu \mathrm{m}^{2}$ ) and larger mean FV1,000 area size in the peripheral region; however, these differences did not reach statistical significance. Details of these comparisons are presented in Table 4 and (Figures 3E-H).

\section{Correlations Between OCTA Parameters and BCVA}

To identifying those parameters most strongly associated with BCVA and thus of potential prognostic or diagnostic utility, we conducted Pearson correlation analysis (Table 5). There were strong negative correlations between BCVA and macular DVP-VLD in patients with acute VKH. While at convalescent stage, macular SVP-VPD and parameters in CC have a stronger correlation with BCVA. Stepwise multiple linear regression analysis demonstrated that macular DVP-VLD and macular CCVPD were the best predictive factors for BCVA in the acute and convalescent VKH groups (Table 6).
TABLE 5 | Correlation of best-corrected visual acuity with the parameters of VKH patients in acute phase and convalescent phase.

\begin{tabular}{|c|c|c|c|c|}
\hline \multirow[t]{2}{*}{ BCVA (LogMAR) } & \multicolumn{2}{|c|}{ Acute phase } & \multicolumn{2}{|c|}{ Convalescent phase } \\
\hline & $r$ & $p$-value & $r$ & $p$-value \\
\hline \multicolumn{5}{|l|}{ SVP-VPD } \\
\hline Macular & 0.298 & 0.201 & $-0.445^{\star}$ & 0.014 \\
\hline Whole FOV & -0.143 & 0.549 & -0.340 & 0.066 \\
\hline Peripapillary & 0.049 & 0.837 & 0.076 & 0.692 \\
\hline \multicolumn{5}{|l|}{ SVP-VLD } \\
\hline Macular & 0.381 & 0.098 & -0.210 & 0.265 \\
\hline Whole FOV & -0.089 & 0.709 & -0.240 & 0.201 \\
\hline Peripapillary & 0.193 & 0.415 & 0.275 & 0.141 \\
\hline \multicolumn{5}{|l|}{ DVP-VPD } \\
\hline Macular & -0.367 & 0.112 & 0.088 & 0.644 \\
\hline Whole FOV & -0.132 & 0.580 & -0.353 & 0.055 \\
\hline \multicolumn{5}{|l|}{ DVP-VLD } \\
\hline Macular & $-0.500^{\star}$ & 0.025 & 0.060 & 0.752 \\
\hline Whole FOV & -0.154 & 0.516 & -0.303 & 0.104 \\
\hline \multicolumn{5}{|l|}{ CC-VPD } \\
\hline Macular & -0.243 & 0.301 & $-0.441^{\star}$ & 0.015 \\
\hline Whole FOV & -0.370 & 0.108 & $-0.373^{\star}$ & 0.042 \\
\hline \multicolumn{5}{|l|}{ Macular CC-FV } \\
\hline Flow void area (\%) & 0.235 & 0.319 & $0.414^{\star}$ & 0.023 \\
\hline \multicolumn{5}{|l|}{ Peripheral CC-FV } \\
\hline Flow void area (\%) & 0.149 & 0.532 & $0.365^{\star}$ & 0.047 \\
\hline
\end{tabular}

r, Pearson correlation coefficient. VPD, vascular perfusion density; VLD, vascular length density; SVP, superficial vascular plexus; DVP, deep vascular plexus; CC, choriocapillaris; FV, flow voids; FOV, field of view. ${ }^{*} p<0.05$.

\section{DISCUSSION}

Vogt-Koyanagi-Harada disease is a multisystemic autoimmune disorder that attacks tissues containing melanin, and thus mainly damages the RPE and choroid in the eyes (2). Acute uveitic phase is characterized by acute, bilateral and diffuse uveitis; hyperemia and edema of the optic disk; choroidal thickening and serous retinal detachment $(33,34)$. As the disease progresses, there can be signs of SGF or RPE clumping/migration, DalenFuchs nodules or multifocal chorioretinal atrophy (34). Multiple imaging modalities have been used to examine the choroidal features in different stages of VKH disease. On fluorescein fundus angiography (FFA), the retina exhibited disseminated spotted choroidal hyperfluorescence and choroidal hypofluorescence in both acute and chronic uveitic stages (35), while ICGA showed hypofluorescent dark dots during initial acute VKH uveitis episodes that usually resolved after therapy (36). Laser speckle flowgraphy (LSFG), which can non-invasively visualize the hemodynamics of choroidal circulation, revealed inflammationrelated impairment in choroidal blood flow velocity at the macula that was again improved by systemic corticosteroid therapy (37).

Optical coherence tomography angiography is a new noninvasive imaging technique that can detect the movement of erythrocytes within blood vessels (9). Compared to spectraldomain OCT (SD-OCT), swept-source OCT (SS-OCT) is able 
TABLE 6 | Stepwise Multiple Linear Regression Analysis for the Predictors of LogMAR BCVA.

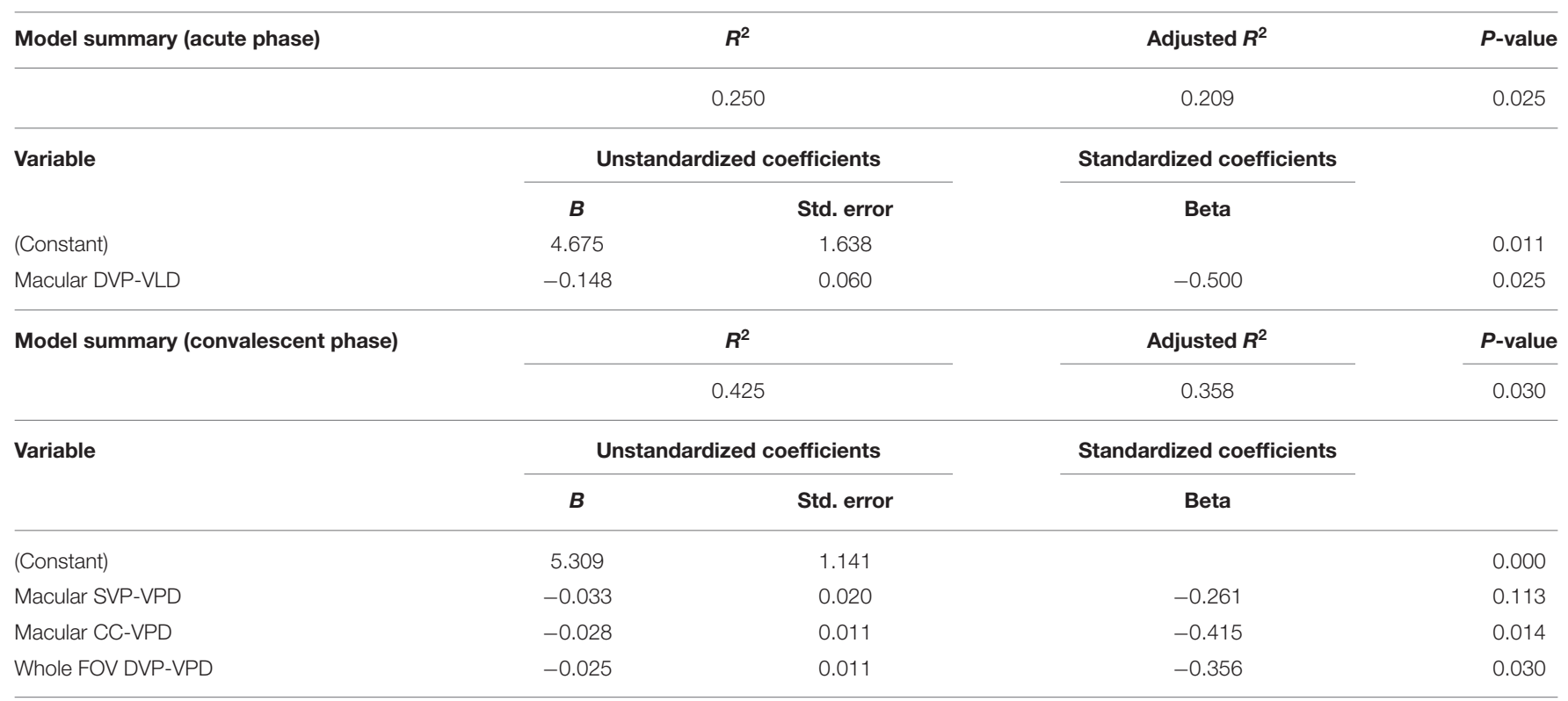

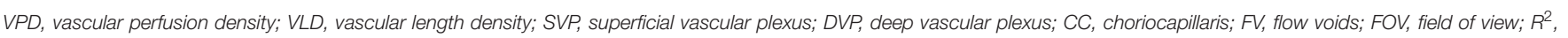
regression coefficient; Std. error, standard error.

to incorporate longer wavelengths $(1,040$ to $1,060 \mathrm{~nm})$ and to simultaneously provide images of the vitreous, retina, and choroid (9), yielding high-resolution near-complete images of choroidal anatomy and CC perfusion (38). Therefore, SS-OCTA is a better imaging modality tool for detailed chorioretinal angiography than SD-OCTA.

Flow voids appear as dark foci in the OCTA CC slab and multiple studies have demonstrated that most FV revealed by OCTA correspond to hypofluorescent spots on ICGA images $(19,24,39-42)$ and hyporeflective spots on enhanced-depth imaging (EDI)-OCT (43). Aggarwal et al. reported that the CC FV on OCTA images reflected true CC ischemia instead of a shadowing effect from overlying subretinal fluid and RPE detachment as was observed in central serous chorioretinopathy (19). Thus, hypoperfusion spots in CC may be attributed to choroidal granulomas $(24,39)$ or blood flow perturbance caused by choroidal vessel inflammation (39).

In our study, VKH disease patients demonstrated increased $\mathrm{CC}-\mathrm{FV}$ and FV average size during the acute phase but not the convalescent phase, consistent with previous studies reporting that systemic corticosteroids can mitigate FV (17, 22, 24, 40). Indeed, the CC-FV observed in convalescent-stage $\mathrm{VKH}$ disease patients did not differ significantly from those measured in HCs. Further, the CC-FV area fraction and CC-VPD correlated strongly with logMAR BCVA during the convalescent phase. The CC-VPD was also dramatically reduced during the acute stage but fully restored during the convalescent phase, in contrast to several previous reports $(21-23,44)$. We speculate that this reflects effective recovery of CC blood flow after appropriate treatment. These positive correlations strongly suggest that choroidal granulomas and persistent inflammation contribute to the vision impairment in $\mathrm{VKH}$ disease. Consistent with this notion, Wintergerst et al. reported a case of acute $\mathrm{VKH}$ syndrome with massively hypoperfusion in Sattler's layer on OCTA that fully resolved after 4 weeks of treatment concomitant with improved BCVA (20). However, that study was the first to use SS-OCTA for evaluation of CC abnormalities in VKH, so differences in detection device, retinal region, and (or) retinal stratification may have accounted for changes between preand post-treatment.

Luo et al. divided acute VKH patients into two subgroups (1 and 2) according to choroid flow area (21), while in the current study we did not observe the subgroup 1 showing significantly enhanced capillary signals, possibly due to the small sample size. Moreover, perfusion density in the CC and flow void size were correlated with visual acuity in our study, suggesting that higher perfusion density [or greater flow area as described by Luo et al. (21)] predict better visual outcome.

In addition to CC, OCTA also allows visualization and quantitative analyses of retinal vessel morphology and perfusion in SVP and DVP slabs. Many researches have revealed a decrease of vascular density when inflammation occurs in fundus, such as Behçet Uveitis (10) and retinal vasculitis (45). Correspondingly, SVP-VPD was markedly reduced in acute VKH disease patients, and a similar trend was also found for the DVP (but did not reach significance). Previous studies have reported similar findings for the SVP, while others have found greater changes in the DVP $(16,23)$. Different stratification methods may account for these discrepancies. Compared to VPD, the alteration in VLD was relative mild. The SVP-VLD of convalescent VKH disease patients was slightly increased compared to HCs. Though these values were not statistically significant, based on the fact that a minority of VKH patients will develop macular choroidal neovascularization (46), the difference suggests that 
flow through small blood vessels and capillaries may increase during the convalescent phase as a compensatory mechanism against ischemia caused by inflammation.

A classic manifestation of acute $\mathrm{VKH}$ disease revealed by fundus photography is hyperemia and by FFA, hyperfluorescence around the optic disc (35). Surprisingly, however, we found that the peripapillary region VPD was lower among acute VKH disease patients. Therefore, we suggest that inflammation may also affect small vessels near the optic disc. Indeed, peripapillary blood flow is also reduced in patients with optic neuritis (4749). However, we cannot exclude possible papilledema as eyes afflicted with optic neuritis also showed decreased blood flow (48). Further studies are awaited.

In the current study, logMAR BCVA was strongly correlated with DVP-VLD despite the insignificant changes in DVP among acute $\mathrm{VKH}$ disease patients compared to convalescent patients and controls. This may due to enhanced importance of the DVP for outer retina perfusion when the choroid is ischemic (23). Pearson correlation analysis showed parameters in CC slab correlate best with BCVA and stepwise multiple linear regression analysis demonstrated that macular CCVPD were the best predictive factors for BCVA in the convalescent VKH group. The results indicated a more significant role of CC slab than SVP/DVP in convalescent $\mathrm{VKH}$ disease.

Limitations of this study include the modest sample size and grouping based mainly on manifestations in the eye rather than more extensive clinical features. Further, patients received different treatment regimens. Manual correction for inaccuracies of retinochoroidal layer segmentation in images acquired using PLEX 9000 OCTA (performed in this study) may lead to errors in vessel density and perfusion calculations.

To the best of our knowledge, this is the first study to apply a SS-OCTA device to capture widefield $15 \times 9 \mathrm{~mm}^{2}$ images revealing microvasculature changes associated with $\mathrm{VKH}$ disease. The large imaging field avoid potential mistakes of montage pictures thus allow for better simultaneously observation of macular and peripapillary areas in fundus thereby mitigating the many potential errors inherent in analysis of montages. We explore the peripapillary microvasculature for the first time. Besides, we also calculate the total length of blood vessel per unit area (vessel length density) in the retina of VKH patients, which

\section{REFERENCES}

1. Du L, Kijlstra A, Yang P. Vogt-Koyanagi-Harada disease: novel insights into pathophysiology, diagnosis and treatment. Prog Retin Eye Res. (2016) 52:84-111. doi: 10.1016/j.preteyeres.2016.02.002

2. Greco A, Fusconi M, Gallo A, Turchetta R, Marinelli C, Macri GF, et al. Vogt-Koyanagi-Harada syndrome. Autoimmun Rev. (2013) 12:10338. doi: 10.1016/j.autrev.2013.01.004

3. Bordaberry MF. Vogt-Koyanagi-Harada disease: diagnosis and treatments update. Curr Opin Ophthalmol. (2010) 21:4305. doi: 10.1097/ICU.0b013e32833eb78c

4. Fang W, Yang P. Vogt-koyanagi-harada syndrome. Curr Eye Res. (2008) 33:517-23. doi: 10.1080/02713680802233968 offers a fresh perspective to explore vascular changes in $\mathrm{VKH}$ disease. Further studies are needed to confirm our results.

In conclusion, the quantifiable and layer-specific characteristics of wider field SS-OCAT enable better evaluation of the microvasculature features of $\mathrm{VKH}$ disease and identification of superior markers for prognosis and treatment guidance.

\section{DATA AVAILABILITY STATEMENT}

The raw data supporting the conclusions of this article will be made available by the authors, without undue reservation.

\section{ETHICS STATEMENT}

The studies involving human participants were reviewed and approved by Guangzhou, China 2019KYPJ127, Medical Ethics Committee of Zhongshan Ophthalmic Center, Sun Yat-sen University (Guangzhou, China). Written informed consent to participate in this study was provided by the participants' legal guardian/next of kin.

\section{AUTHOR CONTRIBUTIONS}

WC, JY, FW, XY, and HZ contributed to conception and design of the study. XH, LS, and JL conducted the OCTA imaging. XY, HZ, $P X$, and GW processed the images and organized the database. $\mathrm{XY}, \mathrm{HZ}$, and FL performed the statistical analysis. XY, HZ, CY, and $\mathrm{YH}$ wrote the first draft of the manuscript. JY and WC wrote sections of the manuscript. All authors contributed to manuscript revision, read, and approved the submitted version.

\section{FUNDING}

This work was supported by National Natural Science Foundation of China (no. 82070950) and Science and Technology Program of Guangzhou of WC (no. 201804010415).

\section{ACKNOWLEDGMENTS}

We would like to acknowledge the assistance of Guandi Chen at Zhongshan Ophthalmic Center for her contribution to assisting in examining the patients and providing images.

5. Moorthy RS, Inomata H, Rao NA. Vogt-Koyanagi-Harada syndrome. Surv Ophthalmol. (1995) 39:265-92. doi: 10.1016/S0039-6257(05)80105-5

6. Magliyah MS, Al-Fakhri AS, Al-Dhibi HA. Proliferative retinopathy as a feature of Vogt Koyanagi Harada Disease: a report of two cases. BMC Ophthalmol. (2020) 20:470. doi: 10.1186/s12886-020-01736-y

7. Ipek SC, Ayhan Z, Emre S, Saatci AO. Favorable clinical outcome with intravitreal aflibercept treatment in a case with bilateral choroidal neovascular membrane and quiescent Vogt-Koyanagi-Harada syndrome. GMS Ophthalmol Cases. (2020) 10:Doc23. doi: 10.3205/oc000150

8. Agrawal R, Li LK, Nakhate V, Khandelwal N, Mahendradas P. Choroidal vascularity index in Vogt-Koyanagi-Harada disease: an EDI-OCT derived tool for monitoring disease progression. Transl Vis Sci Technol. (2016) 5:7. doi: $10.1167 /$ tvst.5.4.7 
9. Lains I, Wang JC, Cui Y, Katz R, Vingopoulos F, Staurenghi G, et al. Retinal applications of swept source optical coherence tomography (OCT) and optical coherence tomography angiography (OCTA). Prog Retin Eye Res. (2021) 2021:100951. doi: 10.1016/j.preteyeres.2021.100951

10. Khairallah M, Abroug N, Khochtali S, Mahmoud A, Jelliti B, Coscas G, et al. UVEITIS. Retina. (2017) 37:1678-91. doi: 10.1097/IAE.0000000000001418

11. Toto L, Borrelli E, Di Antonio L, Carpineto P, Mastropasqua R. Retinal vascular. Plexuses' changes in dry age-related macular degeneration, evaluated by means of optical coherence tomography angiography. Retina. (2016) 36:1566-72. doi: 10.1097/IAE.0000000000000962

12. Arrigo A, Aragona E, Bordato A, Amato A, Borghesan F, Bandello F, et al. Quantitative OCTA parameter variations after treatment of macular neovascularization secondary to age-related macular degeneration. Retina. (2020) 41:1463-9. doi: 10.1097/IAE.0000000000003065

13. Bae K, Bang SK, Kang SW, Kim ES Yu SY. Gap in capillary perfusion and burden of treatment in branch retinal vein occlusion: a wide-field optical coherence tomography angiography study. Retina. (2020) 41:122735. doi: 10.1097/IAE.0000000000003006

14. Kawai K, Uji A, Murakami T, Kadomoto S, Oritani Y, Dodo Y, et al. Image evaluation of artificial intelligence supported optical coherence tomography angiography imaging using OCT-HS100 device in diabetic retinopathy. Retina. (2020) 41:1730-8. doi: 10.1097/IAE.0000000000003101

15. Borrelli E, Sacconi R, Brambati M, Bandello F, Querques G. In vivo rotational three-dimensional OCTA analysis of microaneurysms in the human diabetic retina. Sci Rep. (2019) 9:16789. doi: 10.1038/s41598-019-53357-1

16. Karaca I, Yilmaz SG, Afrashi F, Nalçaci S. Assessment of macular capillary perfusion in patients with inactive Vogt-Koyanagi-Harada disease: an optical coherence tomography angiography study. Graefes Arch Clin Exp Ophthalmol. (2020) 258:1181-90. doi: 10.1007/s00417-020-04676-x

17. Vingopoulos F, Cui Y, Katz R, Le R, Zhu Y, Wang JC, et al. Widefield sweptsource OCTA in Vogt-Koyanagi-Harada disease. Ophthalmic Surg Lasers Imaging Retina. (2020) 51:407-12. doi: 10.3928/23258160-20200702-06

18. Fan S, Lin D, Hu J, Cao J, Wu K, Li Y, et al. Evaluation of microvasculature alterations in convalescent Vogt-Koyanagi-Harada disease using optical coherence tomography angiography. Eye (Lond). (2020). doi: 10.1038/s41433-020-01210-5

19. Aggarwal K, Agarwal A, Deokar A, Mahajan S, Singh R, Bansal R, et al. Distinguishing features of acute Vogt-Koyanagi-Harada disease and acute central serous chorioretinopathy on optical coherence tomography angiography and en face optical coherence tomography imaging. JOphthalmic Inflamm Infect. (2017) 7:3. doi: 10.1186/s12348-016-0122-z

20. Wintergerst MWM, Herrmann P, Finger RP. Optical coherence tomography angiography for evaluation of Sattler's layer in VogtKoyanagi-Harada disease. Ophthalmic Surg Lasers Imaging Retina. (2018) 49:639-42. doi: 10.3928/23258160-20180803-14

21. Luo K, Cai H, Hu Y, Jin C, Gan X, Deng Y, et al. Distinguishing microvasculature features of vogt-koyanagi-harada in patients in acute and convalescent phases using optical coherence tomography angiography. Ocul Immunol Inflamm. (2020) 29:465-71. doi: 10.1080/09273948.2019. 1695856

22. Khan HA, Iqbal F, Shahzad MA, Khan QA, Rashid F, Sharjeel M, et al. Textural properties of choriocapillaris on OCTA in healed inflammatory choriocapillaropathies. Ophthalmic Surg Lasers Imaging Retina. (2019) 50:566-72. doi: 10.3928/23258160-20190905-05

23. Liang A, Zhao C, Jia S, Gao F, Han X, Pei M, et al. Retinal microcirculation defects on OCTA correlate with active inflammation and vision in Vogt-Koyanagi-Harada disease. Ocul Immunol Inflamm. (2020) 2020:17. doi: 10.1080/09273948.2020.1751212

24. Aggarwal K, Agarwal A, Mahajan S, Invernizzi A, Mandadi SKR, Singh R, et al. The role of optical coherence tomography angiography in the diagnosis and management of acute Vogt-Koyanagi-Harada disease. Ocul Immunol Inflamm. (2018) 26:142-53. doi: 10.1080/09273948.2016.1195001

25. Read RW, Holland GN, Rao NA, Tabbara KF, Ohno S, Arellanes-Garcia L, et al. Revised diagnostic criteria for Vogt-Koyanagi-Harada disease: report of an international committee on nomenclature. Am J Ophthalmol. (2001) 131:647-52. doi: 10.1016/S0002-9394(01)00925-4

26. Zheng F, Chua J, Ke M, Tan B, Yu M, Hu Q, et al. Quantitative OCT angiography of the retinal microvasculature and choriocapillaris in highly myopic eyes with myopic macular degeneration. $\mathrm{Br} J$ Ophthalmol. (2021). doi: 10.1136/bjophthalmol-2020-317632

27. Hong J, Ke M, Tan B, Lau A, Wong D, Yao X, et al. Effect of vessel enhancement filters on the repeatability of measurements obtained from widefield swept-source optical coherence tomography angiography. Sci Rep. (2020) 10:22179. doi: 10.1038/s41598-020-79281-3

28. Ji YS, Alagorie AR, Byon I, Sadda SR. Impact of scan tilt on quantitative assessments using optical coherence tomography angiography. Transl Vis Sci Technol. (2020) 9:46. doi: 10.1167/tvst.9.7.46

29. Ledesma-Gil G, Fernández-Avellaneda P, Spaide RF. Swept source optical coherence tomography angiography imaging of the choriocapillaris. Retina. (2021) 41:https://journals.lww.com/retinajournal/toc/2021/070001373-8. doi: 10.1097/IAE.0000000000003109

30. Borrelli E, Uji A, Toto L, Viggiano P, Evangelista F, Mastropasqua R. In vivo mapping of the choriocapillaris in healthy eyes: a widefield swept-source OCT angiography study. Ophthalmol Retina. (2019) 3:97984. doi: 10.1016/j.oret.2019.05.026

31. Uji A, Balasubramanian S, Lei J, Baghdasaryan E, Al-Sheikh M, Sadda SR. Impact of multiple en face image averaging on quantitative assessment from optical coherence tomography angiography images. Ophthalmology. (2017) 124:944-52. doi: 10.1016/j.ophtha.2017.02.006

32. Dhiman R, Chawla R, Azad SV, Kumar P, Gupta V, Kumar A, et al. Peripapillary retinal and choroidal perfusion in nonarteritic ischemic optic neuropathy using optical coherence tomography angiography. Optom Vis Sci. (2020) 97:583-90. doi: 10.1097/OPX.0000000000001550

33. Sakata VM, da Silva FT, Hirata CE, de Carvalho JF, Yamamoto JH. Diagnosis and classification of Vogt-Koyanagi-Harada disease. Autoimmun Rev. (2014) 13:550-5. doi: 10.1016/j.autrev.2014.01.023

34. Yang P, Zhong Y, Du L, Chi W, Chen L, Zhang R, et al. Development and evaluation of diagnostic criteria for Vogt-Koyanagi-Harada disease. JAMA Ophthalmol. (2018) 136:1025-31. doi: 10.1001/jamaophthalmol.2018.2664

35. Arellanes-Garcia L, Hernandez-Barrios M, Fromow-Guerra J, Cervantes-Fanning P. Fluorescein fundus angiographic findings in Vogt-Koyanagi-Harada syndrome. Int Ophthalmol. (2007) 27:155-61. doi: 10.1007/s10792-006-9027-4

36. Herbort CP, Mantovani A, Bouchenaki N. Indocyanine green angiography in Vogt-Koyanagi-Harada disease: angiographic signs and utility in patient follow-up. Int Ophthalmol. (2007) 27:173-82. doi: 10.1007/s10792-007-9060-y

37. Hirose S, Saito W, Yoshida K, Saito M, Dong Z, Namba K, et al. Elevated choroidal blood flow velocity during systemic corticosteroid therapy in Vogt-Koyanagi-Harada disease. Acta Ophthalmol. (2008) 86:9027. doi: $10.1111 / j .1755-3768.2008 .01384 . x$

38. Chu Z, Weinstein JE, Wang RK, Pepple KL. Quantitative analysis of the choriocapillaris in uveitis using en face swept-source optical coherence tomography angiography. Am J Ophthalmol. (2020) 218:1727. doi: 10.1016/j.ajo.2020.05.006

39. Pichi F, Smith SD, Neri P, Woodstock E, Hay S, Parrulli S, et al. Choroidal granulomas visualized by swept-source optical coherence tomography angiography. Retina (Philadelphia, Pa). (2020) 41:602-9. doi: 10.1097/IAE.0000000000002864

40. Erba S, Govetto A, Scialdone A, Casalino G. Role of optical coherence tomography angiography in Vogt-Koyanagi-Harada disease. GMS Ophthalmol Cases. (2021) 11:Doc06. doi: 10.3205/oc000179

41. Cennamo G, Romano MR, Iovino C, de Crecchio G, Cennamo G. Optical coherence tomography angiography in incomplete acute Vogt-Koyanagi-Harada disease. Int J Ophthalmol. (2017) 10:661-2. doi: 10.18240/ijo.2017.04.27

42. Giannakouras P, Andreanos K, Giavi B, Diagourtas A. Optical coherence tomography angiography: employing a novel technique for investigation in Vogt-Koyanagi-Harada disease. Case Rep Ophthalmol. (2017) 8:3629. doi: $10.1159 / 000477611$

43. Pichi F, Aggarwal K, Neri P, Salvetti P, Lembo A, Nucci $\mathrm{P}$, et al. Choroidal biomarkers. Indian J Ophthalmol. (2018) 66:1716-26. doi: 10.4103/ijo.IJO_893_18

44. Liang A, Jia S, Gao F, Han X, Pei M, Qu Y, et al. Decrease of choriocapillary vascular density measured by optical coherence tomography angiography in Vogt-Koyanagi-Harada disease. Graefes Arch Clin Exp Ophthalmol. (2021). doi: 10.1007/s00417-021-05238-5. [Epub ahead of print]. 
45. Tian M, Tappeiner C, Zinkernagel MS, Huf W, Wolf S, Munk MR. Evaluation of vascular changes in intermediate uveitis and retinal vasculitis using swept-source wide-field optical coherence tomography angiography. $\mathrm{BrJ}$ Ophthalmol. (2019) 103:1289-95. doi: 10.1136/bjophthalmol-2018-313078

46. Yang P, Ye Z, Xu J, Du L, Zhou Q, Qi J, et al. Macular abnormalities in Vogt-Koyanagi-Harada disease. Ocul Immunol Inflamm. (2019) 27:1195202. doi: 10.1080/09273948.2019.1624781

47. Lee GI, Park KA, Oh SY, Min JH, Kim BJ. Peripapillary and parafoveal microvascular changes in eyes with optic neuritis and their fellow eyes measured by optical coherence tomography angiography: an exploratory study. Acta Ophthalmol. (2020) 99:288-298. doi: 10.1111/ aos. 14577

48. Fard MA, Jalili J, Sahraiyan A, Khojasteh H, Hejazi M, Ritch R, et al. Optical coherence tomography angiography in optic disc swelling. Am J Ophthalmol. (2018) 191:116-23. doi: 10.1016/j.ajo.2018. 04.017

49. Fard MA, Yadegari S, Ghahvechian H, Moghimi S, Soltani-Moghaddam R, Subramanian PS. Optical Coherence tomography angiography of a pale optic disc in demyelinating optic neuritis and ischemic optic neuropathy. J Neuroophthalmol. (2019) 39:339-44. doi: 10.1097/WNO.0000000000 000775
Conflict of Interest: The authors declare that the research was conducted in the absence of any commercial or financial relationships that could be construed as a potential conflict of interest.

The reviewer XL declared a shared affiliation with one of the authors, HZ, to the handling editor at time of review.

Publisher's Note: All claims expressed in this article are solely those of the authors and do not necessarily represent those of their affiliated organizations, or those of the publisher, the editors and the reviewers. Any product that may be evaluated in this article, or claim that may be made by its manufacturer, is not guaranteed or endorsed by the publisher.

Copyright (c) 2021 Ye, Zhang, Xiao, Wang, Hu, Yan, Li, Hu, Su, Luo, Yuan, Wen and Chi. This is an open-access article distributed under the terms of the Creative Commons Attribution License (CC BY). The use, distribution or reproduction in other forums is permitted, provided the original author(s) and the copyright owner(s) are credited and that the original publication in this journal is cited, in accordance with accepted academic practice. No use, distribution or reproduction is permitted which does not comply with these terms. 